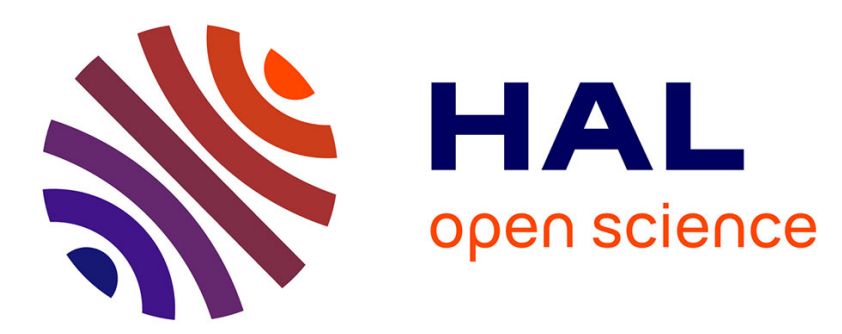

\title{
A New Approach to Design an Interactive System for Molecular Analysis
}

\author{
Mouna Essabbah, Samir Otmane, Joan Hérisson, Malik Mallem
}

\section{To cite this version:}

Mouna Essabbah, Samir Otmane, Joan Hérisson, Malik Mallem. A New Approach to Design an Interactive System for Molecular Analysis. 13th International Conference on Human-Computer Interaction (HCI International 2009), Jul 2009, San Diego, CA, United States. pp.713-722, 10.1007/978-3-64202583-9_77. hal-00407168

\section{HAL Id: hal-00407168 https://hal.science/hal-00407168}

Submitted on 23 Jul 2009

HAL is a multi-disciplinary open access archive for the deposit and dissemination of scientific research documents, whether they are published or not. The documents may come from teaching and research institutions in France or abroad, or from public or private research centers.
L'archive ouverte pluridisciplinaire HAL, est destinée au dépôt et à la diffusion de documents scientifiques de niveau recherche, publiés ou non, émanant des établissements d'enseignement et de recherche français ou étrangers, des laboratoires publics ou privés. 


\title{
A New Approach to Design an Interactive System for Molecular Analysis
}

\author{
Mouna Essabbah ${ }^{1}$, Samir Otmane ${ }^{1}$, Joan Hérisson ${ }^{2}$ and Malik Mallem ${ }^{1}$, \\ ${ }^{1}$ IBISC laboratory, CNRS, University of Evry, Evry, France \\ ${ }^{2}$ Epigenomics Project, Genopole, Evry, France \\ \{mouna.essabbah, samir.otmane, malik.mallem\}@ibisc.fr \\ joan.herisson@epigenomique.genopole.fr
}

\begin{abstract}
The rapid evolution of molecule's imaging and observation's techniques has caused a growing interest in studying molecular structures. Naturally, scientists have turned to simulation and 3D modeling in order to better understand biological phenomena. Thus, several 3D modeling systems have emerged. Some of these systems are dedicated to 3D visualization, and others are interested in 3D handling. However, we observed that these systems use classical 3D interaction techniques, frequently used in virtual reality (VR). On the other hand, the biological environment is very complex and binding as well. Thus, to remain faithful to the constraintes of the environment and be closer to natural behavior of molecules, we have tried to propose a 3D manipulation adapted to the domain, a bio-supervised 3D manipulation.
\end{abstract}

Keywords: 3D Manipulation, Complex Systems, Biological Constraints, Adaptability, Bio-supervisor.

\section{Introduction}

Understand and interpret the DNA's spatial organization has become one of the greatest challenge of recent molecular and structural biology. Biologists are trying to identify the link between molecule's 3D architecture and its functional aspect. So, 3D molecular modeling systems tend to realism and more credibility, and that both on the 3D model and in terms of interaction with this model.

Previously, we have established a global and more credible 3D model of DNA [1], however this will not be detailed in this paper. Nevertheless, the realism of 3D modeling systems will also be performed through the way to interact with the model.

Actually, user interfaces have become increasingly complex: at the visual level, by the graphic richness, 3D environments, animations, etc., and the interactive level, by the wide range of devices and complexity of tasks. Each of these points is the subject of many researches. Indeed, the needs change from one application to another, but the majority remains based on the development of static applications, often limited to support usual interaction techniques. In the case of 3D environments, the management of interactions is even more difficult because user devices are no longer limited to a 
2 Mouna Essabbah1, Samir Otmane1, Joan Hérisson2 and Malik Mallem1,

mouse and keyboard, and tasks are no longer as simple as pointing and clicking. Therefore, the design of 3D applications must take into account the management of interactions at the same level as the actions undertaken by the system. The interaction is no longer an independent bloc in the system architecture, because it must be able to adapt to the environment in which it will be integrated. The modality of interaction defined by a document is not necessarily the same as the applications context.

In our research, we develop an assistance system for molecular analysis while proposing a more credible and global genome's representation. Thus, the handled object is not simple. Moreover, beyond the 3D environment constraints, it is subjected to other very strong constraints, those of physics and biology. It seems obvious that the interactive activity of this application must be adapted to the domain.

In this paper, we present a new approach to design bio-supervised 3D manipulation techniques that is aware of the environment. First, we define the context as well as the raised issues, particularly, the constraints of 3D and biological environments. Then we describe previous work in the assistance for molecular analysis field. The last section will include a description of the proposed approach, where we explain the global architecture; afterward we denote the interest of each component. We conclude this paper with some discussions and future work.

\section{Context and Issues}

\subsection{D Environment Constraints}

The choice of representation and metaphors do not respect a set of fixed rules. If the choice of representation is realistic, as in our case, interactions will also work with the constraints that we find in the real world (i.e. biological environment).

In contrast, if the choice of representation is moving towards a non-realistic environment, simple metaphors will be used to represent complex tasks. The choice of these metaphors is dependent of devices that we decided to use and consistency that we want to keep for the global application. For a given task, there is non-fixed number of possible interaction's metaphors. To take into account these constraints, we orient our platform to enable the creation of various elements of the application.

The devices used will be very varied depending on the application, ranging from simple keyboard and mouse to more specific devices such as data gloves, tracking head, etc. This diversity is due to the complexity of the task to realize in the virtual environment that can claim a many degrees of freedom. Furthermore, the choice of devices becomes more delicate when the tasks are heterogeneous, it must be done through compromise among all tasks. This involves the development of interaction techniques to achieve the task with a device, although it is less suitable (Eg trackball or ray-casting that allow interaction for the selection and manipulation of 3D objects with a simple $2 \mathrm{D}$ device). 


\subsection{Biological Environment Constraints}

The interest of a part of our research is to obtain a 3D model of the molecule's structure, which is closer to the reality. Hence, we rely on natural elements, as the molecule in its environment. Indeed, researches around the genome's organization have identified some biophysical data and biological models, each one represent a part of this spatial organization. We are looking particularly for DNA's organization, at the chromatin level.

Architecural Constraints: Hence, the physical data that we have been based on are: the chromatin's diameter (which is of 30nm [2]), the chromatin's persistence length - an intrinsic feature of the chromatin fiber that represents the rigidity of the chromatin, and that is $150 \mathrm{~nm}$ for the chromatin in vitro [3] -, the confined volume of in which the DNA evolve (the cell nucleus and the chrmosome's territory [4]), and finally, the curvature energy of the chromatin.

Functional Constraints: The architectural constraints represent only the physical aspect of the chromatin, disregarding its functional aspect. Thus, we are interested in biological models. Each model has different physico-chemical and biological implications that will affect the molecule's 3D model. Among the models that will be exploited, we quote: The 3C method (Capturing Chromosome Conformation) [5], the location of transcription factories [6], the formation of loops [7] and the solenoid principle [8].

The challenge that we have fixed is to provide an assistance system for molecular analysis while proposing a credible and global genome's representation. After identifying data and models to exploit, we have worked on building a simple first structure of the molecule that is only based on biophysical constraints. The four biophysical constraints that we have mentioned above, allow us to establish a simple chromatin's 3D model, which is a "worm-like" model based on a sequence of identical cylinders (Fig. 1 shows the conceptual model).
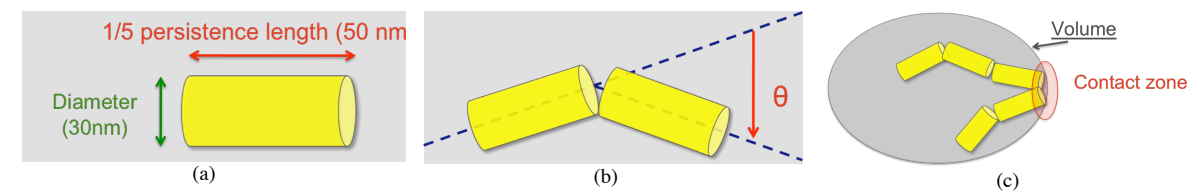

Fig. 1. (a) Basic cylinder: The diameter $=$ chromatin's diameter $(30 \mathrm{~nm})$ and the length $=1 / 5$ chromatin's persistence length $(150 \mathrm{~nm})$, (b) Angle determining the curvature energy, (c) The confined volume in the cell's nucleus

\section{Assistance to DNA's Analysis: Related Work}

Initially scientists were interested in sequence analysis for the study of its rich syntax. Gradually (between 1984 and 1987) molecular biology lived a fulgurating progress of its technical means, which leads to automation and an increasingly advanced and refined miniaturization. On the other hand, genomics allowed nowadays a global and complete approach for sequence analysis. It is no longer limited to the molecules study, but it also includes the study of the relationships between these elements, 
which causes the dynamic aspect of the whole. DNA can be represented by its textual sequence and also by its spatial distribution. The main interest for the threedimensional visualization of this molecule is the study of genes. But this can be done simply by textual analysis of the sequence. However, the 3D model showed that two patterns of the genome might have spatial similarities, and have very different textual sequences. It is also possible, thanks to the 3D model, to seek a pattern throughout the molecule not only by its sequence but also by its spatial form. This could deduct the influence of the entire genome in certain areas and vice versa. On the other hand, DNA spatial visualization provides an opportunity to study its interaction with other molecules, especially proteins. More generally, 3D visualization provides an overall view of the studied molecule. It allows modeling phenomenon or simulates a biological mechanism.

Currently, molecular modeling is an essential research area. It helps scientists to develop new drugs against diseases in general and particularly serious ones such as AIDS and cancer. This sector assists also the genomes analysis. As the interest that was accorded is extremely important, many researchers became specialized in molecular modeling.

The assistance in analyzing the DNA's spatial structure was done in two stages: first by a completely automated method and then a more interactive approach.

\subsection{Automatic Approach}

DNA's 3D visualization is based mainly on two approaches. First, the observatory approach consists in reproducing the molecule's 3D model from crystallographic data, which is not fairly well accepted in the case of DNA, because current techniques for the experimental study of DNA's 3D structure (crystallography, cryo-electron microscopy, AFM microscopy, etc.) present many limitations (size, molecule's deformations, etc..) and are very costly in time and price. Dispates, several threedimensional molecular viewers, simple and freely available, have emerged (gOpenMol, MoleKel, ViewMol, MolMol, PyMol, RasMol (Fig. 2), etc.). It is possible to have more examples in this study that we have conducted [9]. Besides, most modelisation concerns especially proteins thanks to a great $3 \mathrm{D}$ data bank ("PDB” format, Protein Data Bank).

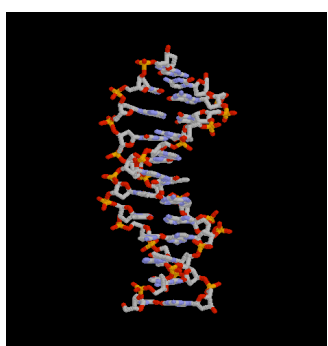

Fig. 2. RasMol: free software for viewing PDB files

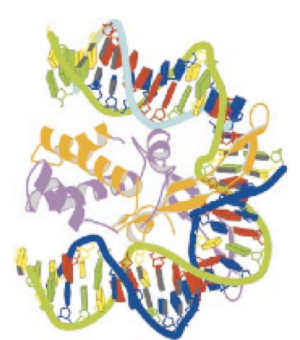

Fig. 3. 3DNA: local DNA's 3D modeling by a predictive method

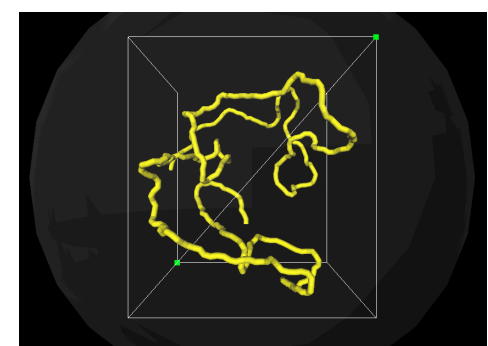

Fig. 4. DNA's 3D model based on biophysical data 
That's why 3D modeling techniques have been developed. Hence, the predictive approach aim to predict approximately the DNA's 3D structure from its textual sequence (Fig. 3) thanks to spatial conformation model of DNA (obtained by statistical methods on small fragments of DNA), which is considerably easier to implement, cheaper and faster than the experimental methods in vitro.

In our case, a part of the research was to propose a new DNA's 3D modeling (Fig. 4) based on biophysical data (described in section 2.2). However, the backtracking algorithm that we use is also costly in computing time, in order to cover a large search space [1].

Therefore, the automatic processes of DNA's 3D modeling and visualization must overcome an important difficulty to reach relevant results, which is the computation time of research algorithms. Hence, the DNA's 3D modeling and analysis assisted by multi-modal VR-technology is a relevent alternative to enhance the result.

In the past, the most interesting molecular 3D modeling/visualizing systems have been either too large or too complex to model in real time, but now, many molecular 3D simulation programs run efficiently on parallel computers. Thus, a new opportunity was offered to biologists who may now have an interactive control on those applications during their execution.

\subsection{Interactive Approach}

Gradually the visualization interest no longer stops at the molecules observation, but it extends to the structure analysis and functionality interpretation of molecules by their forms. Moreover, the limitations of conventional 3D modeling algorithms and the new possibilities offered by Virtual Reality (VR), brought some research teams to look on problems related to interactive and immersive molecular modeling [10]. Early works in this area have focused on identification of technical needs and technological limitations. It was research in an area totally unknown by biology, at the time.

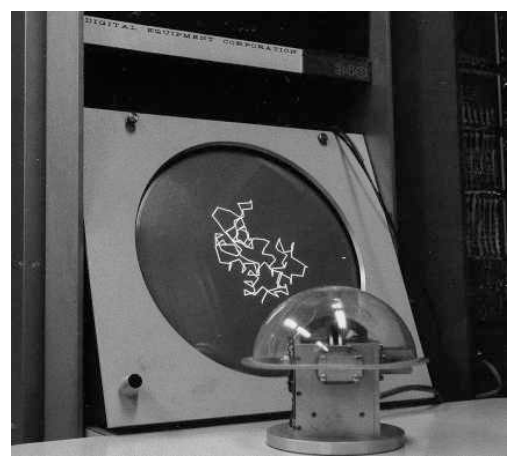

Fig. 5. The display terminal shows the molecule structure in wireframe fashion

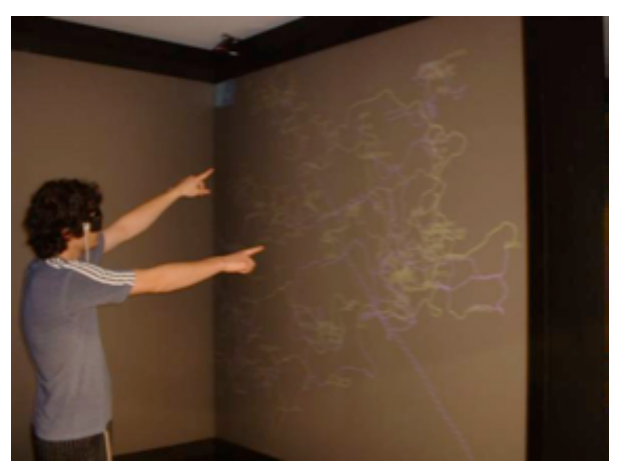

Fig. 6. Stereoscopic visualization of chromosomes and immersive navigation with ADN-Viewer 
Cyrus Levinthal, at MIT, built the first system for the interactive display of molecular structures in the mid-1960s. This system allowed a user to study interaction between atoms and the online manipulation of molecular structures. The speed and orientation of a molecular structure can be controlled by a globe-shaped device (a kind of trackball) (Fig. 5 shows the VR interface used)[11]. Moreover, it was possible to select from a menu, choose an object, or zoom into important parts of the molecule using a light pen on the computer display.

Subsequently, new interactive viewers appear in order to study the molecule dynamics through its three-dimensional structure. These specific applications offer more complex modeling like molecules functionality. The immersive system AMMPVis uses a virtual environment for collaborative molecular modeling [12]. It offers to biologists and chemists the possibility of manipulating molecular models through a natural gesture. It allows receiving and displaying real-time molecular dynamics simulation results. It allows also adapted views sharing and provides support for local and remote collaborative research.

The highly immersive molecular modeling (HIMM) environment is built from computer aided molecular modeling tools (already done) and virtual reality (VR) engines [13]. The proposed architecture tends to provide with minimal effort a flexible HIMM environment.

Other research has highlighted the importance of using VR for education, combining immersive environment (efficient visual representation) and haptic devises (interactive manipulation) to offer a good instructional aid for students [14].

Another system, ADN-Viewer [15] is particularly interested in DNA's spatial distribution. The software offers 3D reconstruction of DNA's structure through several 3D representations. It also provides the opportunity to explore chromosomes' structures thanks to stereoscopic visualization (Fig. 6).

In the same principle of stereoscopic navigation, a stereographic table has been built by Marchese and al. [16] to support molecular visualization.

Scientists have developed many others interactive molecular visualization software even with collaborative technologies $[17,18]$. However, they are often limited in only a part of the interface with the virtual reality (either navigation, or selection, or manipulation, etc.) and this is insufficient to really explore the ability of multimodal interaction in VR to improve the molecular analysis.

\section{A Hybrid and Multimodal Approach}

\subsection{Hybrid Approach}

Our approach aims to combine the contribution of multimodal rendering and the experts' knowledge in certain phases of the study. In fact, multimodal vitual environments succed better tha single-sensory technologies in creating a sense of presence [19]. In allowing the user to interact earlier in the process rather than post or by setting the single system, we hope to reduce the computing time and also the risks of wrong 3D models. More Specifically, the protocol that we develop is user-centred. It describes a cycle that alternates successively using the automatic and the interactive 
approach of modeling by the user's command (illustrated in Fig. 7). The first step is related to the automatic procedure of modeling, and will generate an initial 3D structure, with the geometrical computing engine. Then, in the immersive environment, the biologist will be able to improve the 3D model in the configurations that seems interesting through visual, auditory and haptic renderings. During this stage, the user has, as reference, a set of biological models at its disposal. This first step will quickly reduce the search space of possible conformations, being based on the expert's capacity of analysing 3D patterns and his knowledge of the specific molecule and biological models. Finally, the expert can choose to restart automatic generation of 3D model -locally or globally-, but taking into account the current changes.

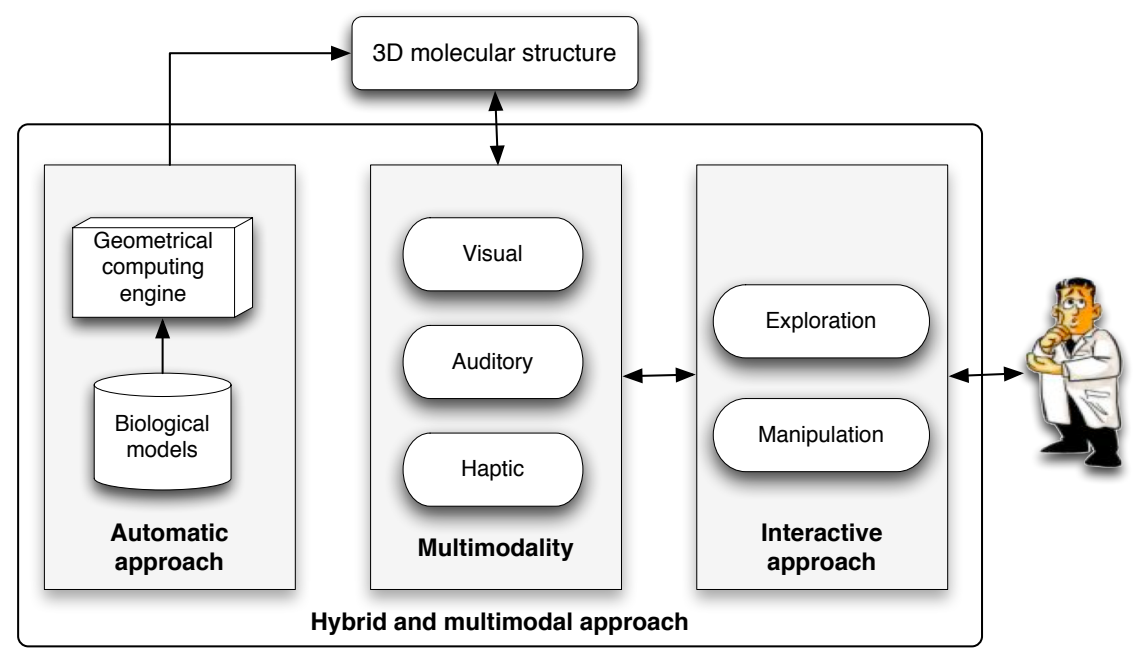

Fig. 7. Hybrid approach for the assistance to DNA's analysis

\subsection{Hardware Architecture}

The hardware architecture of our molecular modeling environment is based on a semi-immersive VR/AR multimodal platform called EVR@ ${ }^{1}$ (see Fig. 9). It permits stereoscopic display, wireless hand/head gesture tracking and force feedback. We have ART optical tracking system composed of two ARTTrack1 infrared cameras, two wireless Flystick, one head marker and one hand marker. We also have a 6D force feedback device SPIDAR-G ${ }^{2}$ [20] and 5DT Data Gloves. Each device is associated to a specific server, which is accessed via the C++ VRPN library [21] by clients. The interactivity between the user and the VE is done by using Virtools ${ }^{\mathrm{TM}} 4.0$ [22] as a front-end.

\footnotetext{
${ }^{1}$ http://evra.ibisc.univ-evry.fr

${ }^{2}$ Space Interface Device for Artificial Reality
} 


\subsection{Software Architecture}

To take into account these constraints, we orient our approach to a modular architecture (Fig. 8). The modular approach, beside the advantage of reusability, is also able to modify or adapt more easily an application. We will refine the components through the design phase.

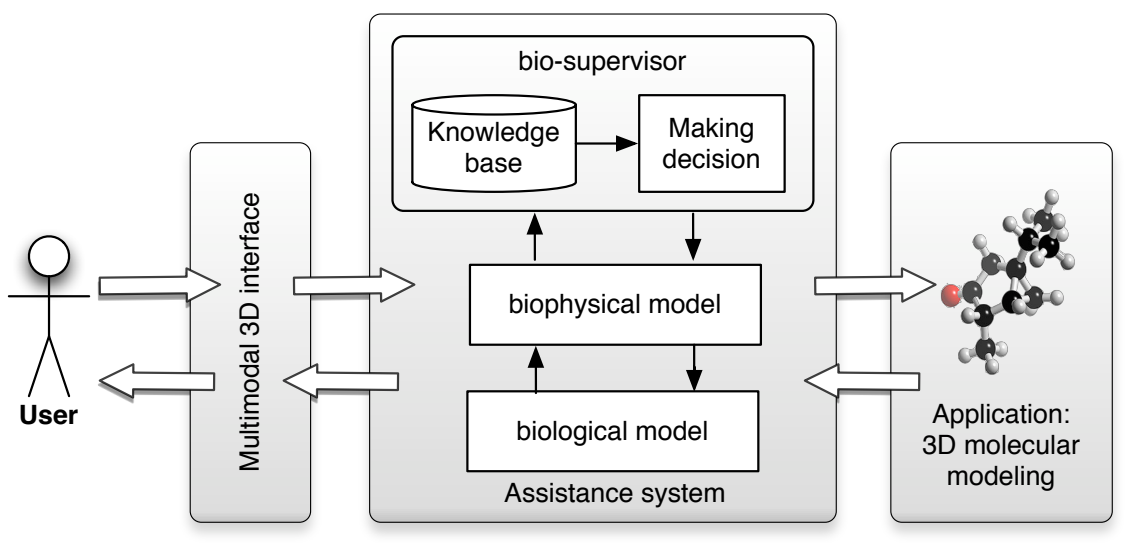

Fig. 8. An assistance system for molecular analysis based on bio-supervised 3D interaction.

The architecture of a classical 3D interaction is often divided into two parts: the user and the different devices, and the application, which try to communicate with the user through these devices. So, we propose the design of a new 3D interaction paradigm, using what we call a bio-supervisor, by adding this new component between the user and the VR application. The supervisor concept has already been used for 3D multimodal interaction by Bouyer and al. [23] in order to make the 3D interaction more intuitive for the user, but without taking into account the domain (ie. the environment).

In our case, the supervisor role will be to control the user's actions according to the biological constraints that we have already mentioned. When interacting, if the user exceeds any of these constraints, the bio-supervisor must let him know while limiting its action. Therefore, a multimodal interaction must be set up so that the user becomes aware of the limits imposed by the supervisor by a visual, auditory and haptic feedback. For instance, the task to perform is to curve the chromatin (molecule), however, the chromatin's energy restrains the curvature, imposing a physical limit. In this case, the user can get a visual and auditory feedback through a classical workstation, however, he can exceed the limits and continue to twist the chromatin whereas the 3D model does not follow the command. Thus, there is a contradiction between user's action and the 3D model rendering. In contrast, in addition to the known benefits (a real 3D rendering, a human scale, an immersion, etc.), a virtual reality platform (see Fig. 9) will provide coherence between the user's action and the limits imposed on the 3D model. Hence, through a multimodal feedback system (visual, auditory, haptic, etc.), the user will become aware of the 3D model limit, but it also will feel it and will be restricted on it. 


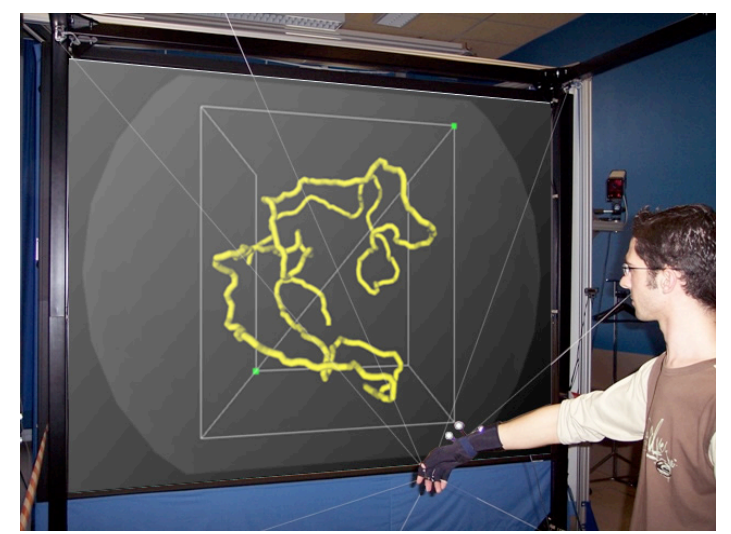

Fig. 9. Handling a 3D molecule through our semi-immersive virtual/augmented reality platform: Evr@.

\section{Conclusion}

We have described in this paper a new approach to design 3D interaction techniques context-aware, that is an important goal in our work in order to propose a 3D molecular modeling system, more natural and intuitive. Our objective is to set up a framework of 3D interaction favorable to the complex and constraining biological environment. Indeed, through the $3 \mathrm{D}$ interaction, we want to provide an active assistance for the manipulation and the analysis of DNA in a very intuitive way. Moreover, we can take advantage of the multi-modality to offer to the user the maximum of information that could assist him, for example, use the force feedback through haptic devices in order to represent more naturally the constraints and limits of biology. The next work is the evaluation of the proposed assistance system with subjective and objective assessments.

\section{References}

1. Essabbah, M., Hérisson, J., Otmane, S., Mallem, M.: Towards a biophysical 3D model of the DNA. In: 1th IEEE International Workshops on Image Processing Theory, Tools and Applications, pp. 1-6, Sousse (2008).

2. Philip J. J. Robinson, Louise Fairall, Van A. T. Huynh, and Rhodes D.: EM measurements define the dimensions of the "30-nm" chromatin fiber: Evidence for a compact, interdigitated structure, Proc Natl Acad Sci. USA, April 25; 103(17): 6506-6511, (2006).

3. Bystricky K., Heun P., Gehlen L., Langowski J., and Gasser S. M.: Long-range compaction and flexibility of interphace chromatin in budding yeast analyzed by high-resolution imagining techniques. Proc. Natl. Acad. Sci.USA, 101(47): 16495-16500, (2004). 
4. Cremer T., Cremer M., Dietzel S., Muller S., Solovei I. and Fakan S.: Chromosome territories - a functional nuclear landscape, Current Opinion in Cell Biology Vol. 18, 307316, (2006).

5. Dekker J., Rippe K., Dekker M. and Kleckner N.: Capturing Chromosome Conformation, Science 295, 1306, (2002).

6. Cook P.R., Predicting three-dimensional genome structure from transcriptional activity, nature genetics Vol. 32, 347-352, (2002).

7. Marenduzzo D., Faro-Trindade I. and Cook P.R.: What are the molecular ties that maintain genomic loops?, TRENDS in Genetics Vol. 23, No. 3, 126-133, (2006).

8. Képès F. and Vaillant C.: Transcription-BasedSolenoidalModel of Chromosomes, Complexus Vol. 1, 171-180, (2004).

9. Essabbah M., Otmane S. and Mallem M.: 3D molecular modeling: from theory to applications. In IEEE Conference on Human System Interaction (HSI'08), pp 350-355, Krakow (Poland), (2008).

10.Marchese F.T.: Molecular Visualization at the Interface, In Trends in Interactive Visualisation / Advanced Information and Knowledge Processing Series, pp 251-268, Springer-Verlag, London, (2009).

11.Levinthal C.: Molecular model-building by computer. Sci Am 214 (6), pp 42-52, (1966).

12.Chastine J.W., Brooks J.C, Zhu Y., Owen G., Harrison RW., and WeberIT.: AMMP-Vis: a collaborative virtual environment for molecular modeling. In Proceedings of the ACM Symposium on Virtual Reality Software and Technology (Monterey, CA, USA, VRST '05. ACM Press, New York, NY, pp 8-15, (2005).

13.Drees RC, Pleiss J, Schmid RD, Roller D: Integrating molecular modeling tools and virtual reality engines: an architecture for a highly immersive molecular modeling (HIMM) environment. In: Proceedings of the computer graphics international. IEEE Computer Society, Washington, DC, pp. 391-392, (1998).

14.Sato M., Liu X., Murayama J., Akahane K., Isshiki M.: A Haptic Virtual Environment for Molecular Chemistry Education. T. Edutainment 1, pp 28-39, (2008).

15.Hérisson J. and Gherbi R.: Model-Based Prediction of the 3D Trajectory of Huge DNA Sequences Interactive Visualization and Exploration, 2nd IEEE International Symposium on Bioinformatics and Bioengineering (BIBE’01), pp 263-270, (2001).

16.Marchese FT: A stereographic table for biomolecular visualization. In: Proceedings of the sixth international conference on information visualization: IV'02. IEEE Computer Society, Washington, DC, pp. 603 - 607, (2002).

17.Marchese FT, Brajkovska N.: Fostering asynchronous collaborative visualization. In: Proceedings of the 11th international conference information visualization: IV'07. IEEE Computer Society, Washington, DC, pp. 185 -190, (2007).

18.Su S, Loftin R, Chen D, Fang Y, Lin CH Distributed collaborative virtual environment: PaulingWorld. In: Proceedings of 10th international conference on artificial reality and telexistence, pp. 112-117, (2000).

19.Hecht, D., Reiner, M., and Halevy, G.: Multimodal virtual environments: response times, attention, and presence. Presence: Teleoper. Virtual Environ. 15, pp 515-523, (2006).

20.Kim et al: Development of tension based haptic interface and possibility of its application to virtual reality, In Proceedings of the ACM symposium on Virtual reality software and technology, pp. 199-205, (2000).

21.Taylor et al.: VRPN: a device-independent, network-transparent VR peripheral system. VRST '01: Proceedings of the ACM symposium on Virtual reality software and technology, pp. $55-61,(2001)$.

22.Dassault Systemes, http://www.virtools.com/.

23.Bouyer G., Bourdot P.: Supervision of $3 \mathrm{~d}$ multimodal rendering for protein-protein virtual docking. In Eurographics Symposium on Virtual Environments (EGVE), volume 28, pp 49$56,(2008)$. 